neuerer Zeit die Beamten der kaiserlichen Sternwarte gelangten, weisen auf dieselbe Thatsache hin. Obschon nun die dort geleiteten Untersuclungen und Reobachtungen bis jetzt noch nicht vollständig bekannt sind, so giebt doch Herr Tournet, unter Mitwirkung der Herren Lambert und Rassimier, die Schlussfolgerungen, bei der Wiederaufnahme in Lyon und dessen Vorstädten; während in Sauvage auf den Höhen von Tararae, einer Bergreihe, welche das Becken der Loire und der Rhone trennt, Ozon reichlich rorhanden gewesen, waren in Lyon 1-2mal im Monat kaum Spuren nachzuweisen.

Nun wird man sich erinnern, dass oft behauptet wurde, das Auftreten der Cholera treffe zusammen mit dem Verschwinden des Ozons in der Luft. Das Beispiel von Lyon trifft mit dieser Behauptung nicbt überein, da die Stadt auch ohne Ozon der Cholera nicht unterworfen ist.

(Chemical news [Ausland 1867]). Dr. Löhr.

\title{
Ueber Thiergifte
}

referirte Herr Dr. Köhle $r$ (in der Sitzung des Naturw. Vereins f. Sachsen u. Thüringen am 16. Oct. 1867). Ein solches, nämlich das den Sala$\mathrm{m}$ ander $\mathrm{n}$ eigenthümliche, wurde 1866 von $\mathrm{Z}$ alesky in Tübingen isolirt und $\mathrm{S}$ amandarin genannt.

Es ist stickstoffhaltig, den Pflanzenalkaloïden analog zusammengesetzt und durch Phosphormolybdänsäure fällbar wie diese.

Ueber ein zweites Thiergift, der $\mathrm{Cobra}$ di $\mathrm{Capello}$ angebörig, hat Prof. If altford in Melbourne zwar nicht chemische, jedoch wichtige morphologische Mittheilungen gemacht. Aus seinen Beobachtungen geht hervor, dass durch das in die Wunde gelangende Secret der genannten Schlange, das Blut mit keimfähiger organischer Materie inficirt wird, welche sich unter Aufnahme gewisser Stoffe des Blutplasmas und des Sauerstoffs zu Millionen und aber Millionen Zellen vermehrt.

Indem sonach dem Blute wesentliche Bestandtheile sowohl, als auch der bei der Circulation durch die Lungen aufgenommene Sauerstoff entzogen werden, entartet dasselbe und gehen die Gebissenen asphyctisch zu Gruade.

Schliesslich macht $\mathrm{Hr}$. Köhler auf die nevesten Untersuchungen über die phosphorhaltigen organischen Stofle, welche von H o p pe-Se yler's Schülern angestellt wurden, aufmerksam, wonach nicht aller Phosphor als Protagon im Thierkörper (im Blute, Eigelb) enthalten ist, sondern noch eine zweite, gleichfalls phosphorhaitige Substanz, das $L$ e ith in darin vorkommt.

(Zcitschr. f. d. ges. Naturw. Oct. 1867.' S. 420). H. Ludwig.

\section{Vergifteter' Weizen.}

In Langenbielau liess sich eine unbekannte Frau 6 Metzen Weizen bei dem dortigen Müller gegen Mebl umtauschen. Der Weizen wurde unter die zu vermahlenden Vorräthe gemischt und das Mehl bei Gelegenheit des Osterfestes an sehr viele ärmere Leute verkauft. Nach dem Genusse der daraus bereiteten Speisen und Gebäcke zeigten sick bei allen Betheiligten Symptome von Vergiftung und mehr als 100 Personen erkrankten, doch ist kein Todesfall zu beklagen gewesen. Von 13 Kühǫn, die mit der Kleie gefüttert worden waren, fielen 8 sebr bald, bei den übrigen war wenig Aussicht auf Erhaltung vorhanden.

Arch d. Pharm, CLXXXIV. Bds, 1, u. 2. Hft. 
Die chemische Untersuchung des Mehles ergab einen starken Zinkgehalt. (Mühlhäuser Anzeiger Nr. 73. Mai 1867). Die Bunzlauer Zeitung führt an, dass dem Mehle Arsenik beigemischt gewesen sei. Wahrscheinlich war dem allgemeinen Gebrauchegemäss der Weizen zur Erböhung der Keimfähigkeit mit Zinkvitriol gebeizt, was oft auch mit Kupfervitriol geschieht, wie ich selbst vielfach in Mühlhausen in Thüringen zu erfahren Gelegenheit gebabt habe. - Die Leipziger Apotheker Zeitung (Nr. 20. 16. Mai 1867) schlägt zu dem gleichen Zwecke Eisenvitriol vor.

Dr. Reich.

\section{Stearinkerzen mit Arsenik.}

Seit mehren Jahren kommen Stearinkerzen in den Handel, welche sich durch ihre alabasterartige Weisse auszeichnen und deshalb ein vielbegehrter Artikel sind. Erleuchtet man ein Zimmer mit einer grössern Anzahl dieser Kerzen, so nimmt die Luft eine drückende Schwere an, ein eigenthümlicher, scluwach an Knoblauch erinnernder Geruch erfült den ganzen Raum, und es stellen sich eigenthümliche Krankheitssymptome bei den Bewohnern dieser Zimmer cin.

Durch die Krankbeitsfalle, welche eineu kaum zu verkennenden Oliarakter annehmen, argwöhnisch gemacht, schenkte man der Sache mehr Aufmerkamkeit und besonders einem dünnen nebelartigen Hauche, der sich auf vorher sorgfältig geputzten Scheiben, Spiegel und den polirten Flächen der Möbel zeigte. Die chemische Untersuchung dieses feinen Staubes ergab, dass er aus reinem weissen Arsenik bestand, die verlockenden Kerzen waren mit einer nicht unerheblichen Menge Arsenik versetzt. Während des Verbrennens verwandelt sich derselbe in Dampf, der den knoblauchartigen Geruch verbreitet und sich an kältern Gegenständen als Giftstaub sbsetzt. Wie stark die Azwendung des weissen Arseniks in der Kerzenfabrikation war und theilwcise noch ist, geht daraus hervor, dass von London aus nach dem Kontinent centnerweise ein Geheimmittel importirt wird, welches dazu dient, Stearin - und Talglichtern eine blendende Weisse und grosse Härte zu geben. Dies Geheimmittel ist nichts Anderes als höchst fein gepulverter Arsenik. Jede Kerze, welche auf dem Bruche ein mehr schwammiges als krystallinisch festes Gefüge erkennen lässt und beim Verbrennen einen schwachen weissen Rauch ausstösst, ist als verdächtig anzusehen. Eine chemische Analyse muss dann die Anwesenheit des Arseniks constatiren. Es fehlt eigentlich der rechte Ausdruck, dies Verfahren gewissenloser Kerzenfabrikanten $z u$ bezeichnen; denn jedenfalls ist doch vorauszusetzen, dass ihnen die Gefährlichkeit des angewandten Materials bekannt ist. Das Leben und die Gesundheit einer grossen Anzabl seiner Mitmenschen auf das Spiel zu setzen, um aus Gewinnsuoht einem unentbehrlichon Artikel durch Beimischung eines der schłecklichsten Gifte grössern Absatz zu verschaffen, ist eine Handlungsweise, die auf das Strengste geabndet werden müsste. Hier und da, z. B. in Köln, sind einzelne Stimmen darüber laut geworden, aber sic sind ungehört verballt; es hilft nicht immer, den Sanitätsbehörden ein "Brutus, schläfst Du?" zuzurufen. Erst wenn das Kind hineingefallen, wird der Brunnen zugedeckt.

(Gothaisches Tageblatt. Mai 1867). Dr. Reich. 